\title{
Proceso y oportunidades de la transferencia del conocimiento desde la psicología comunitaria a las políticas públicas*
}

Process and Opportunities for Knowledge Transfer from Community Psychology to Public Policy

Recibido: 1 de marzo de 2015 | Revisado: 1 junio de 2015 | Aceptado: 1 agosto de 2015

\author{
JAime Alfaro I. ** \\ Universidad del Desarrollo, Santiago, Chile \\ María Pía Martín M.**** \\ Universidad de Chile, Santiago, Chile
}

doi:10.11144/Javeriana.up14-4.potc

Para citar este artículo: Alfaro, J., \& Martín, M. P. (2015). Proceso y oportunidades de la transferencia del conocimiento desde la psicología comunitaria a las políticas públicas. Universitas Psychologica, 14(4), 1347-1358. http://dx.doi.org/10.11144/Javeriana. up14-4.potc

* Artículo de investigación. Este trabajo se origina en el Proyecto FONDECYT de Investigación Regular $\mathrm{N}^{\circ} 1150938$ "La configuración del campo técnico del psicólogo en la política social: análisis de la formulación e implementación de programas sociales con componente psicosocial

** Doctor en Ciencias Sociales y de la Educación de la Universidad de Girona. Investigador Docente Facultad de Psicología. Director Centro de Estudios en Bienestar y Convivencia Social Universidad del Desarrollo. Correo electrónico: jalfaro@udd.cl

*** Doctora en Políticas Públicas del Instituto de Gobierno y Políticas Públicas (IGOP) de la Universidad Autónoma de Barcelona. Académica del Departamento de Ingeniería de la Universidad de Chile. Directora de Estudios de Caso del Magíster en Gestión y Políticas Públicas Correo electrónico: mpmartin@dii.uchile.cl

\section{RESUMEN}

La literatura reporta una creciente incorporación y participación de la psicología comunitaria en políticas públicas, que genera obstáculos, tensiones y posibilidades. Cobra relevancia académica y profesional analizar los antecedentes sobre transferencia de conocimiento desde la psicología comunitaria hacia las políticas públicas. Se revisa y expone literatura respecto de las condiciones que afectan el vínculo entre la producción académica y las respuestas a los problemas sociales desde la perspectiva de las deliberative politcs, del proceso de encuentro y de la lucha de interpretaciones de actores como factor de cambio de las políticas públicas. Desde la psicología comunitaria, se analiza la complejidad de transferencia del conocimiento hacia las políticas públicas, concluyendo con recomendaciones para potenciar la contribución y comprender las tensiones reportadas.

Palabras clave

psicología comunitaria; políticas públicas; transferencia de conocimiento; políticas públicas deliberativas; intervención comunitaria

\footnotetext{
A B S T R A C T

The literature reports the growing incorporation and participation of community psychology on public policies, and considering the obstacles and tensions in this relationship as reported by literature,-it is both academically and professionally relevant to review the knowledge transfer from the academia to the policy making process. We review a body of literature regarding the link between academic production and the response to social problems, emphasizing the deliberative politics approach, with a description of stakeholders' confluent and conflicting interpretations as a factor of change for public policies. We also review the literature regarding the complexity of knowledge transfer from community psychology to public policy, as reported by this discipline, concluding with recommendations for enhancing its contribution and to understand the tensions reported. Keywords community psychology; public policy; knowledge transfer; deliberative politics; community intervention
} 
La investigación académica en el ámbito de las políticas públicas enfrenta la demanda, así como el desafío, de responder e influir con instrumentos y técnicas en las decisiones que adoptan los formuladores de políticas (policymakers). No obstante, la relevancia de conocer las condicionantes, alternativas y logros que se dan en la relación entre academia y políticas públicas, el vínculo ha sido poco explorado, siendo necesario incrementar la comprensión sobre esta dinámica, de manera que la gestión del conocimiento contribuya a un mejor uso y aplicación de conocimientos para la resolución y, en general, la innovación en el abordaje de problemáticas sociales.

Avanzar en la sistematización y análisis del estudio de esta relación resulta particularmente relevante para la psicología comunitaria, en el marco de su creciente incorporación y participación de sus profesionales en las diversas fases de proceso de políticas públicas y sociales, según se ha reportado en las últimas décadas, desde distintos centros académicos y diversas regiones (Alfaro, 2012; Ornelas, Vargas-Moniz, \& Madeira, 2012; Rodríguez, 2009), así como también de manera particular en el contexto de las discusiones y debates respecto de la complejidad, tensiones y riesgos que entraña esta relación (Freitas, 2000; Krause, 2002; Lapalma \& De Lellis, 2012). Adquiriendo relevancia de esta manera el estudio y análisis del proceso de transferencia de conocimiento académico a las políticas públicas en el ámbito de la psicología comunitaria.

Desde la literatura sobre políticas públicas, se ha venido desarrollando una línea que propicia un mejor vínculo entre la producción académica de las ciencias sociales en general y de la psicología comunitaria en particular, y que remite a la línea de deliberative politics (políticas públicas deliberativas). El análisis de políticas públicas en general en América Latina ha estado dominado por las visiones racionalistas provenientes de la economía, principalmente. Sin embargo, la propuesta desde las deliberative politics enfatiza que las respuestas a los problemas sociales requieren de la participación de las comunidades para definir tanto los problemas públicos como las políticas públicas que se diseñan para abordarlos. De manera que en formulación de políticas (policymaking), las decisiones en las diversas etapas de desarrollo de las políticas y las opciones de los agentes gubernamentales no se relacionan, de manera directa y simple, con bases de conocimiento y no se traducen en productos de decisiones meramente técnicas, sino que involucran procesos de decisiones de múltiples actores que actúan en el tiempo de manera simultánea o secuencial. Se evidencia que el proceso de transferencia de conocimientos hacia las políticas está cruzado por tensiones múltiples que es necesario asumir como condición para la articulación entre el mundo académico y los formuladores de políticas. Con base en lo cual se propone y fundamenta la necesidad de tener en cuenta aspectos institucionales propios de las políticas públicas, en particular considerando aspectos relacionados con las dinámicas de actores sociales y los marcos de ideas y valores que comportan en los mecanismos de participación en las políticas públicas.

En virtud de la importancia que revisten estos procesos para la acción social desde la psicología comunitaria, en este trabajo se revisan y discuten distintas dimensiones relevantes para comprender la configuración y organización de las políticas públicas. Se expone la literatura relativa a las dimensiones que permiten comprender las formas, diversidad y dinámicas de la relación entre conocimiento, desde las nociones de participación contenidas en la psicología comunitaria, y su vinculación con las políticas públicas deliberativas. En particular, se revisan el debate y análisis desarrollados tanto desde los estudios en políticas públicas, que resaltan la participación como elemento central, como desde la psicología comunitaria, respecto de las dimensiones que hacen parte y constituyen la complejidad de la relación, y afectan la posibilidad del proceso de transferencia de conocimiento a las políticas.

\section{Trayectoria, contribución mutua y tensión en la relación entre psicología comunitaria y políticas públicas}

El vínculo entre políticas públicas y psicología comunitaria es una dimensión relevante desde el origen de esta última, enlazada con el objeto y 
propósito de su intervención. Según autores clásicos como Rappaport (1977) y según revisiones más actuales como las de Phillips (2000) y Bishop, Vicary, Browne y Guard (2009), la transformación de las políticas públicas en los años sesenta tuvo incidencia en el origen de la psicología comunitaria. Asimismo, este vínculo se incrementó hacia los años ochenta, y sobre todo los noventa, e incluyó no solo dimensiones como la salud mental, tal como fue en sus inicios, sino que el conjunto de las áreas de la intervención social, a la vez que tuvo presencia en diversas realidades regionales.

La literatura ahonda además en el proceso de contribución e influencia mutua entre estos campos. Al respecto, diversos autores destacan, en primer lugar, los aportes hechos con base en su condición de disciplina científica y, en segundo lugar, los aportes derivados del acervo conceptual y técnico de la psicología comunitaria. Se habrían aportado insumos de conocimiento científico en los distintos niveles o fases del ciclo de las políticas públicas, tales como la formulación, la fundamentación, el diseño, la ejecución, el seguimiento, la evaluación y la fijación de prioridades (Bishop et al., 2009; Ornelas et al., 2012; Phillips, 2000).

Asimismo, también se reporta la incidencia de las políticas públicas sobre el desarrollo de la psicología comunitaria, estableciendo una relación entre el proceso de desarrollo y cambio de las primeras con la evolución de la segunda (Burton, Boyle, Harris, \& Kagan, 2007; Teixeira et al., 2012) y que las orientaciones de acción por implementarse en la comunidad se vincularían con las estrategias de desarrollo del país, especialmente la incorporación de aspectos psicosociales en el diseño y ejecución de políticas sociales, afectando con ello directamente la práctica profesional de los psicólogos comunitarios. Por otra parte, las nociones de empoderamiento y de participación de la psicología comunitaria contribuyen con conocimientos a las deliberative politics. De modo que el empowerment es un proceso que dota a un individuo o colectivo de la capacidad para dirigir el cambio en su vida, con el fin de proporcionar un grado de autonomía y control sobre el mundo que lo rodea (Zimmerman $\&$ Rappaport, 1988). Independientemente de la complejidad de la deliberación pública, la expectativa de los efectos positivos de este tipo de prácticas en los participantes ha allanado el camino para un "giro deliberativo", donde las decisiones sobre definición de problemas públicos, agenda gubernamental y formulación de políticas no las toma un grupo de expertos con base en racionalidades técnicas y económicas. Más bien, suponen la participación de múltiples actores y racionalidades en un contexto donde la complejidad de los problemas públicos requiere un diagnóstico y una definición desde múltiples perspectivas que involucran una variedad de actores y colectivos sociales en un marco de gobernanza participativa, donde al Gobierno le corresponde coordinar y liderar el conjunto de la red para buscar políticas que den soluciones a los problemas públicos (Blanco, 2004; Goodin, 2008). Las críticas a la racionalidad señaladas por Stone (2002), Fischer (2003) y Zittoun (2009) señalan que el interés individual aparece como el elemento determinante del comportamiento de las personas, no reconociendo cómo los sistemas de ideas, significados y los marcos interpretativos impactan la forma en que los actores perciben y modifican sus intereses y desarrollan construcciones conjuntas al interpretar la realidad, generando cambios de políticas.

En este mismo sentido, autores como Shinn (2007) y Ornelas et al. (2012), entre otros, señalan lo fundamental de considerar que las políticas sociales conllevan diferentes visiones o filosofías con incidencia en las posibilidades de las acciones derivadas desde la disciplina. A tal punto que la existencia misma del campo comunitario como escenario de acción se ve afectado por el espacio que las políticas públicas le otorguen (Burton et al., 2007; Teixeira et al., 2012). A la vez, basándose en planteamientos de autores como Marcoux, Angelique y Culley (2009), entre otros, se puede establecer que el proceso de contribución a las políticas públicas, señalado antes, habría generado condiciones, espacios y oportunidades para el desarrollo de quehaceres y experiencias. Así, también habría permitido la puesta en uso de técnicas propias de la disciplina, en diversos campos, en acciones frente a distintas problemáticas, niveles de 
acción, áreas programáticas y estrategias de trabajo que en su conjunto habrían repercutido en la psicología comunitaria, potenciando la diversificación, la innovación y el enriquecimiento de las prácticas profesionales y académicas propias de esta.

No obstante lo anterior, según recogemos de un conjunto de otros reportes, el vínculo entre psicología comunitaria y políticas públicas entraña también tensiones, complejidades y riesgos. Específicamente, se reporta un conjunto de tensiones y desencuentros entre las orientaciones propias de la psicología comunitaria y determinados encuadres de las políticas públicas, que afectan su vocación crítica y su capacidad para generar cambios sociales (Freitas, 2000; Krause, 2002; Lapalma \& De Lellis, 2012; Montero, 2010; Rodríguez, 2009). Esto limitaría la aplicación y uso efectivo de principios técnicos específicos propios de la psicología comunitaria, tales como la generación de participación social, el empoderamiento y el desarrollo de procesos bottomup, en el marco de las políticas (Alfaro, 2011; Alfaro \& Zambrano, 2009; Bishop et al., 2009; Burton et al., 2007; Freitas, 2000; Krause, 2002; Lapalma \& De Lellis, 2012; Montero, 2010; Rodríguez, 2009). El análisis sobre esta situación de tensión y riesgo refiere a la presencia de determinados modelos de políticas públicas, caracterizados por sus formas asistenciales, paliativas, centradas en el déficit o compensatorias, que derivan de concepciones subsidiarias del rol del Estado (Krause, 2002; Rodríguez, 2009; Teixeira et al., 2012).

\section{Procesos de participación, encuentro y lucha de interpretaciones de actores en las políticas públicas deliberativas}

Entender los procesos de formulación de políticas públicas como parte de procesos deliberativos, de conflicto y encuentro de racionalidades, implica una "comprensión política de las políticas" (Nelson, 2001), que rescata el énfasis de los procesos democráticos, participativos y deliberativos (De Leon, 2006; Fischer, 2003). Fischer y Forester (1993) sostienen que en la medida en que hay tensión entre el conocimiento experto y la democracia, y entre la tecnocracia y la participación de los distintos grupos sociales, plantear la conversión del analista de políticas en un científico 'neutral', con la verdad objetiva sobre las políticas, incentivó la consolidación de elites tecnocráticas, alejadas de la política y los ciudadanos. Por su parte, Schön y Rein (1994) se posicionan contra la "racionalidad técnica" del enfoque tecnocrático y a favor de una práctica más interpretativa y crítica respecto a los instrumentos del poder y las situaciones de conflicto y lucha que hay tras las políticas.

Investigadores en políticas públicas como Fischer (2003), Dryzek (2000), Hajer (1993, 2003) y De Leon (2006) señalan que los supuestos y resultados del enfoque basado en la racionalidad exclusivamente técnica de los procesos de formulación e implementación de políticas, desconoce la complejidad de los problemas sociales y transforma los análisis en instrumentales y antidemocráticos. Como sostiene Stone (2002), "el proyecto de racionalidad" implícito al modelo del ciclo secuencial de las políticas (diseño, implementación y evaluación), sigue un modelo racional usado para la toma de decisiones que falla en capturar la esencia del policy making, que es la lucha en torno a las visiones de mundo y al plano simbólico relativo a una política, el cual, como señala esta autora, en esencia se conforma como una "batalla" constante con relación a los criterios para la clasificación, los límites de las categorías y las definiciones que orientan el comportamiento y la enunciación misma de un problema y sus soluciones.

Diversos autores (Fischer, 2003; Hajer, 2003; Sabatier \& Jenkins-Smith, 1993; Schlager, 1999; Rein \& Schön, 1993) plantean que la creación de visiones compartidas (ideas, discursos, significados, visiones de mundo, marcos de interpretación) y la competencia con otras comunidades de opinión constituyen el núcleo fundacional del proceso de políticas (policy making), debiendo estar por tanto en el centro del análisis de políticas, y específicamente en el estudio de su transformación. Incluso, el cambio institucional puede surgir de una modificación cognitiva o normativa en los valores o creencias de los actores, pues la transformación institucional se explica por la inconsistencia de los marcos cognitivos dominantes en la sociedad y la 
deslegitimación de la institución. La emergencia de nuevas ideas pone en duda el esquema cognitivo y puede llevar a un cambio en las respuestas y capacidades institucionales (Schmidt, 2006).

De Leon y Vogenbeck (2007) plantean que además hay que considerar que la objetividad se considera inadecuada para analizar tareas políticas de por sí no objetivas, y donde se juegan valores e ideas, además de intereses. De este planteamiento deriva la apuesta por una mayor participación en el análisis de las políticas públicas (De Leon, 2006; Dryzeck, 2000; Fischer, 2003), que se va a impulsar a través de la propuesta de la deliberación de políticas públicas o "deliberative policy analysis" (Hajer \& Wagenaar, 2003), definido por Hajer como " $a$ varied search for understandings of society to facilitate meaningful and legitimate political actions, agreed upon in mutual interaction to improve our collective quality of life" (2003, p. 191). Esta definición enfatiza la multiplicidad de métodos que se necesita emplear, porque la deliberación es interactiva y la discusión de las soluciones de políticas permite la legitimidad de las soluciones decididas, lo que incluye la vocación por mejorar la calidad de vida de las personas. En un contexto de vacío institucional, de 'sociedad en red', en el cual no existen reglas de conducta uniformes y aceptadas por todos y donde lo 'científico' es parte de esa negociación, la deliberación permite actuar en lógicas de redes y suplir estos vacíos. La propuesta deliberativa se instala en el análisis interpretativo que, según Hajer, puede iluminar mejor el análisis de políticas en contextos de gobernanza con múltiples actores participando en la formulación de políticas públicas.

En esta propuesta, se busca cambiar la relación vertical entre el analista experto (el que tiene el conocimiento) y el ciudadano. En búsqueda de mecanismos más democráticos, el experto debe servir como facilitador del aprendizaje público y del empoderamiento político, asistiendo a los ciudadanos en sus esfuerzos por examinar sus intereses y tomar sus propias decisiones (Fischer, 1998). Ello se ha desarrollado fundamentalmente en los últimos años en la evaluación de políticas y en los procesos de implementación y diseño, buscando además la legitimación de las decisiones públicas.
Hajer y Laws (2006) opinan que la relación entre analistas, policy makers y ciudadanos debe volver a pensarse como un intercambio democrático. Asimismo, según señala Nelson (2001), tanto la experiencia gubernamental como la práctica deben evitar el cientifismo no testado políticamente que ha demostrado ser inútil para procesar y tomar decisiones políticas.

Sin embargo, la participación de los ciudadanos y su rol en el policy making muchas veces se ha criticado por ser demasiado costoso en términos de tiempo o muy problemático, pues mayores actores dificultan la posibilidad de llegar a acuerdos o lograr consensos. También, como señalan Fischer (2003) y De Leon (2006), se privilegian los acuerdos tecnocráticos antes que la participación de ciudadanos sin conocimientos acabados respecto de temas complejos. Los expertos deben tener una relación colaborativa/participativa con los ciudadanos. Ello, porque, como señalan Goodin, Rein y Moran (2006, p. 5), las soluciones meramente técnicas no dan una respuesta adecuada. Es necesaria la persuasión, porque el análisis de políticas públicas no es un cuerpo exacto y organizado de conocimientos, sino más un arte que una ciencia. Apoyándose en los conceptos de Wildavsky (1969), se plantea que el análisis de políticas es normativo y valórico. Al recomendar unas políticas o modos de políticas se opta, y se presenta necesariamente un componente normativo y frecuentemente prescriptivo también. Esto implica que el análisis de políticas públicas también es normativo, orientado a la acción y basado en la persuasión "These apparently commonplace observations -that policy studies is a 'persuasion' that aspires to normatively committed intervention in the world of action-pose powerful challenges for the policy analyst" (Goodin et al., 2006, p. 6).

De tal forma, el modelo deliberativo de políticas públicas extiende el objetivo de análisis más allá del logro de la eficiencia técnica en las instituciones gubernamentales, para incluir las necesidades y los intereses políticos del conjunto de la comunidad. El análisis de políticas públicas busca, según señalan Fischer y Forester (1993), legitimar una mayor participación de los ciudadanos para afianzar la democratización de los procesos de políticas públicas; lo que 
cobra aún más relevancia en un contexto cambiante de policy making, propio de las sociedades modernas, en donde la expertise científica es también parte de procesos de negociación (Hajer, 2003).

En esta misma línea, Hajer (2003) plantea que, ante problemas complejos, en donde la información es ambigua y poco clara, por más que exista información su procesamiento por parte del analista implica una elección sobre cómo analizar, que no es neutral. Según el autor, hay dos opciones, querer la claridad total, que es a lo que juega el racionalismo económico o los neopositivistas y mostrar una 'verdad', o bien hacerse cargo de esa ambigüedad y mostrar los elementos que están a la base de esta. Ello porque, en el debate público lo que prima no es necesariamente el criterio de racionalidad ni la consistencia técnica o científica de la solución propuesta, sino la capacidad de persuasión, característica de la actividad política. Definir los contornos de un problema implica establecer las posibilidades de su resolución. Por tanto, definir el problema es fundamental. El debate se genera sobre las ideas y alternativas para resolver este problema, donde prima quien tiene mayor legitimidad o capacidad de convencer y legitimar sus posiciones ante la ciudadanía y ante el resto de los actores que interactúan sobre el problema. El debate público es característico de la democracia, pues se busca construir mayorías para impulsar una propuesta de política pública. Es en ese juego de poder, de ideas, de construcción del problema y sus soluciones donde la definición del problema como un elemento esencial para comprender el proceso de elaboración de las políticas públicas, pues las políticas no son mecanismos de respuesta a problemas que existen objetivamente, sino que los problemas y sus soluciones son construidos socialmente (Fischer, 2003).

La definición del problema es un foco principal de análisis de una política pública y de comprensión del cambio en ella, en cuanto, como señalan Stone (2002), Fischer y Forester (1993) y Vlassopoulou (1999), los procesos de políticas son una lucha constante acerca de los criterios para la clasificación y definición de problemas, en donde la interpretación subjetiva de experiencias comunes, el marco conceptual en el que se encuadran el análisis y la defi- nición de los problemas y sus soluciones y las ideas que guían y subyacen a cada uno de estos aspectos son cruciales para entender las características y la constitución de los significados compartidos de los actores y de las dinámicas que motivan su actuación y posicionamiento ante la política.

La relevancia de las ideas en políticas públicas, según este enfoque, se relaciona con que estas juegan un papel independiente en el proceso de las políticas, conformándose como el medio fundamental donde se expresan los conflictos políticos, y donde se formula la estructura de relaciones sociales entre los actores y entre estos y su contexto.

Como plantea Stone (2002), la comunidad política está compuesta por ciudadanos que viven en un entramado de interdependencias, lealtades y asociaciones, en las cuales las preferencias individuales no están 'dadas', sino que se van conformando a partir de visiones de mundo contextualizadas e históricamente situadas.

Desde una noción alternativa a esta concepción, Nelson (2001) considera que en la historia de las políticas públicas esta perspectiva se contrapone a concepciones críticas de la primacía de la ciencia sobre otras formas de conocimiento, asignando relevancia y centralidad al papel de las ideas y los juicios de valor en la resolución de los problemas públicos.

Stone (2002), complementariamente a los planteamientos anteriores, considera que el enfoque racionalista y economicista en el análisis del policy making, está basado en un modelo de razonamiento político centrado en un modelo de sociedad de mercado, que ve a la comunidad como una agregación de intereses individuales y no en un modelo de sociedad como comunidad política. Dificultando la comprensión acerca de que la lucha de visiones sobre lo que es de interés público, refleja una disputa sobre la distribución de bienes públicos, que es situada temporalmente y es consistente con las propias luchas individuales, cobrando por tanto relevancia considerar en el análisis de políticas públicas las visiones de mundo en circulación y disputa en la sociedad.

Dicho de manera más específica, desde esta perspectiva, según señala Stone (2002), los procesos de construcción de representaciones colectivas 
y significados sociales son, al mismo tiempo, condicionantes contextuales de las políticas, parte de las dinámicas y procesos internos, y un componente central de estas. De forma tal que los sentidos, sistemas de creencias y los significados en general (y la lucha entre ellos) sobre lo que es de interés público, es lógica y temporalmente anterior a la lucha por la distribución de bienes públicos. Por lo tanto, en el origen y en el análisis de las políticas públicas son centrales las visiones de mundo en circulación y disputa en la sociedad que están a la base de cada uno de sus componentes. Así, como indica Fischer (2003), el proceso de cambio e influencia de las políticas públicas supone crear y controlar sistemas compartidos de significado social, de manera que los resultados intangibles o simbólicos se constituyen en fines tanto o más importantes que los resultados tangibles, como la distribución de bienes y servicios públicos.

Así, de lo señalado hasta ahora respecto de esta perspectiva, se desprende que, como señala Kay (2009), la pieza central del análisis de políticas es la dinámica de mediación simbólica entre los responsables políticos influyentes, los textos de política, y el proceso de ejecución, los instrumentos y recursos materiales empleados para llegar a un acuerdo y establecer significados compartidos. Es decir, asignando centralidad en el estudio de políticas a la relación entre las declaraciones objetivas de formulación y cambio de las políticas y su comprensión subjetiva.

\section{Complejidad y recomendaciones para potenciar la transferencia del conocimiento desde la psicología comunitaria a las políticas públicas}

Respecto de la complejidad del proceso de transferencia de conocimiento desde la psicología comunitaria a las políticas públicas, en primer lugar, es interesante tener en cuenta, como muestra Perkins (1995), que las conexiones entre el contenido de las políticas y la teoría en psicología comunitaria no solo no son directas, sino que normalmente tienden a ser débiles, de forma tal que resulta simplista creer que una mayor disponibilidad y utilización del conocimiento académico por sí solo, y de manera mecánica, mejoraría la influencia y transferencia a las decisiones gubernamentales. En este sentido, es un error el pensar que simplemente cuanto más conocimiento científico se ponga a disposición y se utilice, mejores serán las decisiones del Gobierno. Más bien, la evidencia y la práctica de trabajo en políticas públicas muestra que su formulación es un proceso complejo, en el que participan actores múltiples, posicionados desde distintos intereses, en donde cobra relevancia crucial familiarizarse y conocer las dinámicas, relaciones y procesos de intercambio entre estos agentes participantes y los distintos niveles de organización que concurren en la formulación e implementación de una política -ejecutivo, legislativo y judicial- y en general con los organismos y organizaciones de todo tipo, desde asociaciones barriales y vecinales hasta las agencias federales e incluso internacionales (Perkins,1995). Es decir, más allá de solo disponer de conocimiento académico riguroso, el vector que posibilita incidencia en políticas, se relaciona con hacerse parte de las dinámicas de interacción de los agentes profesionales, decisores y académicos, de manera que el usos de conocimiento o investigación, más que solo apuntar a proveer decisiones específicas, más bien se oriente a influir por vía de modificar el contexto en el que se formula la política y particularmente influir en la gestión de los equilibrios que conllevan las alianzas y coaliciones entre los actores involucrados, afectando las definiciones y proveyendo representaciones (o metáforas) útiles en la reformulación y problematización del discurso dominante (Perkins,1995).

Por otra parte, Phillips (2000) también asume que la relación entre psicología comunitaria y políticas públicas es compleja, en cuanto los vínculos e intercambio entre estos campos están cruzadas por tensiones múltiples, en las cuales tienen un rol relevante concepciones erradas respecto las políticas públicas presentes entre los agentes disciplinares, que no facilitan entender y actuar en consecuencia y en particular dificultan entender que las políticas públicas se construyen como el arte de lo posible, en un marco de restricciones presupuestarias, agendas, presiones de los partidos políticos, etc. No dimensionado 
suficientemente, según señala esta autora, que en estos procesos juegan un rol relevante los marcos institucionales y normativos que delimitan las políticas, así como también lo juegan las dinámicas bidireccionales desde donde estas se construyen, en un proceso jerárquico de lo nacional o federal a lo local, o también desde lo local a los niveles superiores (Phillips, 2000).

De manera que es relevante asumir que las políticas públicas no son una cadena de eventos predecibles, sino por el contrario, ellas se construyen a través del tiempo y con una multitud de procesos decisionales en modificación permanente, que dependen más que de opciones racionales, de la resolución de conflictos entre agentes múltiples participantes y situados en niveles diversos (Phillips, 2000). En un sentido similar, contribuye también el punto de vista de Solarz (2001), pues considera que el obstáculo principal en la relación entre psicología comunitaria y políticas públicas no es contar o no con conocimiento base para buscar influir, sino que lo importante es comprender las tensiones y dificultades de diálogo y mutuo entendimiento que se generan con los formuladores de política.

En este sentido, resulta relevante tener en cuenta, como se recoge de Bishop et al. (2009), que un obstáculo importante en la relación de la psicología comunitaria con las políticas públicas es el temor que tienen los agentes académicos de perder credibilidad científica, así como también autonomía y capacidad crítica; lo que generaría en ellos una actitud ambivalente respecto de participar en la formulación de políticas sociales. Estos autores recomiendan que para resolver esta ambivalencia y potenciar la posibilidad de generar cambio en las políticas públicas, la psicología comunitaria debe concebir que existe la posibilidad de relacionarse con ella desde diversos roles y desde aproximaciones múltiples, pero que independientemente de la forma que finalmente se asuma, lo fundamental es jugar un rol básico de "participante conceptualizador". Específicamente, señalan Bishop et al. (2009) que debemos ampliar la mirada y asumir que la contribución a las políticas públicas puede darse, tanto desde dentro, participando en la formulación e implementación de las políticas, como desde fuera, criticándolas, en el debate político dentro de los círculos de formulación de las políticas (por ejemplo, cuando la participación asume un rol instrumental de técnico experto) o como agentes críticos externos (en el papel de académico o de activistas comunitarios) o como promotor especializado en determinadas temáticas (problemáticas, estrategias, técnicas). Sin embargo, según resaltan estos autores, lo más importante para avanzar en la vinculación y transformación de las políticas es que, independientemente del lugar que se ocupe, no se desconozca la relevancia de ellas y se apunte a deconstruir y problematizarla, centrando el foco en determinar a quienes afecta y establecer los contextos y visiones de mundo de los participantes, en un contexto multidisciplinario. Este rol, aun siendo parte de la asesoría a los grupos decisores implica situar el funcionamiento del grupo en un contexto local, social, histórico y político amplio. Lo que supone un reconocimiento de que los múltiples agentes son parte del problema que se está intentando resolver y de que es necesario un cambio en las relaciones en donde se pretende intervenir, lo que requiere usar métodos adecuados de participación que empoderen y reconozcan diversidad cultural, admitiendo todas las visiones de los participantes, incluida la comunidad (Bishop et al., 2009).

\section{Conclusiones}

Una primera reflexión derivada de lo expuesto es que el modelo lineal y tecnocrático de asesoramiento científico a los gobiernos y de guía para orientar la influencia y transferencia de conocimiento científico en general, y particularmente desde la psicología comunitaria, definitivamente no se produce en los hechos. Los diversos autores analizados en este trabajo, tanto del campo de las políticas públicas, como de la psicología comunitaria, muestran que las corrientes principales de análisis tienen una perspectiva vertical, que entiende el uso de la investigación social como una simple transferencia de conocimiento desde un científico a un formulador de políticas, que acoge resultados de investigaciones y las aplica en consecuencia en el diseño de políticas. 
Esta perspectiva, según se ha señalado desde los análisis del deliberative politics, presenta límites al enfatizar tecnocracias que no son capaces de resolver los problemas complejos de las sociedades actuales, que requieren de mecanismos de participación y gobernanza. Es necesario considerar y valorar esa perspectiva como una posibilidad entre otras para entender y orientar el diálogo entre la academia de la psicología comunitaria y las políticas públicas. Ello aun cuando, tal cual ha sido reportado en los trabajos expuestos, este proceso de diálogo sea normalmente complejo, integrando dinámicas multiactor y multinivel, en el cual la influencia de la investigación en las políticas públicas ocurre a través de redes y comunidades de políticas, incluyendo luchas entre diversas opciones de definición de los contornos de los problemas públicos y las orientaciones y contenidos de las políticas públicas por formular.

Una segunda reflexión que muestra el análisis realizado es que la comunicación entre científicos y políticos es un proceso de resultados inciertos, y de alta complejidad, cobrando relevancia dos aspectos a tener en cuenta. El primero es que existen distintas culturas entre la comunidad de los formuladores de políticas respecto a la de los productores de conocimiento, lo que hace que la comunicación entre ambas comunidades sea difícil. El segundo aspecto que se debe considerar es que los agentes gubernamentales y los investigadores tienen importantes diferencias en términos de valores, prioridades e incentivos. No existen posturas unívocas, por lo que se deben considerar espacios de discusión entre estos. En muchos casos, cada una de las partes utiliza los conocimientos científicos disponibles y fuentes opuestas de información para fortalecer sus posiciones, en el marco de las luchas ideológicas, de valores y orientaciones que están presentes en la relación entre conocimiento y políticas públicas.

Una tercera reflexión que surge de la anterior es que para generar contextos favorables de interacción o interfaz entre científicos y agentes gubernamentales tomadores de decisiones se requiere la construcción de espacios o estructuras de diálogo que permitan las influencias mutuas y el acercamiento entre dos mundos. Efectivamente, hay una relación compleja entre dos lógicas institucionales diferentes, pero en las sociedades actuales los límites entre la ciencia y la política son cada vez menos taxativos y claros, por lo que las relaciones recursivas y no unidireccionales son las que dominan; de manera que las redes de confianza y la generación de espacios de interacción son cruciales y de ellos depende que se de un uso más frecuente de insumos técnicos en la formulación de políticas.

$\mathrm{Al}$ respecto, es particularmente relevante el hecho que para generar influencias se requiere instalar consensos sobre la definición de los problemas sociales de modo de generar cambios graduales en las representaciones y significados de los actores acerca de las causas de ciertos fenómenos sociales. Ello como vía para impactar en la formulación de las políticas. Por lo tanto, los científicos que deseen generar procesos exitosos de relación con los tomadores de decisión de las políticas públicas, requieren de una cierta comprensión sobre cómo funciona el proceso de la política y cómo la experiencia científica se trata por lo general en la política y se integra en los procesos de diálogo, negociación y resolución de conflictos de interés entre los agentes y coaliciones participantes en el contexto de los problemas y soluciones sociales.

Finalmente, en el contexto de transformaciones que caracterizan a las sociedades contemporáneas es necesario destacar la importancia de las políticas públicas basadas en una sólida investigación empírica, en procesos estructurados de consulta entre múltiples actores y de las redes de investigación y de política, así como en la consideración de la participación de los actores sociales en la producción de conocimientos. De manera que el enlace estratégico entre conocimiento y políticas se genere en un contexto donde se puedan confrontar las diferencias y se produzcan acercamientos en torno a la manera como se aborda un problema de política pública. Los procesos de comunicación, diálogo y concertación entre diversos actores constituyen el contexto inicial definido para alcanzar nuevos aportes, tanto en la generación de conocimiento como en el sentido de la acción. 


\section{Referencias}

Alfaro, J. (2011). Psicología comunitaria y políticas sociales: tensiones y complementariedad en el caso del Programa Chile Solidario. Ponencia presentada en la IV Cátedra Internacional Ignacio Martín-Baró: Intervención psicosocial y superación de la pobreza en Chile, Universidad Alberto Hurtado, Santiago de Chile.

Alfaro, J. (2012). Posibilidades y tensiones en la relación entre psicología comunitaria y políticas sociales. En J. Alfaro, A. Sánchez \& A. Zambrano (Eds.), Psicología comunitaria y políticas sociales: reflexiones y experiencias (pp. 45-76). Buenos Aires: Paidós.

Alfaro, J., \& Zambrano, A. (2009). Psicología comunitaria y políticas sociales en Chile. Psicologia $\mathbb{E}$ Sociedade, 21(2), 275-282.

Bishop, B., Vicary, D., Browne, A., \& Guard, N. (2009). Public policy, participation and the third position: The implication of engaging communities on their own terms. American Journal Community Psychology, 43(1-2), 111-121.

Blanco, I. (2004). Polítiques de regeneració urbana en Barcelona. Els casos del Raval y de la Trinitat Nova (Tesis doctoral inédita). Universidad Autónoma de Barcelona, Barcelona.

Burton, M., Boyle, S., Harris, C., \& Kagan, C. (2007). Community psychology in Britain. En S. Reich, M. Riemer, I. Prilleltensky \& M. Montero (Eds.), International community psychology: History and theories (pp. 219-237). Nueva York: Kluwer Academic Press.

De Leon, P. (2006). The historical roots of the field. En M. Moran, M. Rein \& R. E. Goodin (Eds.), The oxford handbook of public policy (pp. 39-57). Oxford: Oxford University Press.

De Leon, P., \& Vogenbeck, D. (2007). The policy sciences at the crossreads. En F. Fischer, G. Millery \& M. S. Sidney (Eds.), Handbook of public policy analysis. Theory, politics and methods (pp. 3-14). Boca Raton, FL: CRC Press.

Dryzek, J. S. (2000). Deliberative democracy and beyond: Liberals, critics, contestations. Oxford: Oxford University Press.

Fischer, Frank (1998) Beyond Empiricism: Policy Inquiry in Post positivist Perspective Policy Studies Journal Volume 26, Issue 1, pages 129-146
Fischer, F. (2003). Reframing public policy. Discursive politics and deliberative practices. Oxford: Oxford University Press.

Fischer, F., \& Forester, J. (Eds.). (1993). The argumentative turn in policy analysis and planning. Durham: Duke University Press.

Freitas, M. (2000). Voices from the south: The construction of Brazilian community social psychology. Journal of Community \& Applied Social Psychology, 10(4), 315-326.

Goodin, R. E. (2008). Innovating democracy: Democratic theory and practice after the deliberative turn. Oxford: Oxford University Press.

Goodin, R., Rein, M., \& Moran, M. (2006). The public and its policies. En M. Moran, M. Rein \& R. E. Goodin (Eds.), The Oxford handbook of public policy (pp. 3-35). Oxford: Oxford University Press.

Hajer, M. (1993). Discourse coalitions and the institutionalisation of practice. The case of acid rain in Britain. En F. Fischer \& J. Forester (Eds.), The argumentative turn in policy and planning (pp. 43. 76). Durham: Duke University Press.

Hajer, M. (2003). A frame in the fields: Policymaking and the reinvention of politics. En M. Hajer \& H. Wanegaar (Eds.), Deliberative policy analysis. Understanding governance in the network society (pp. 88-112). Cambridge: Cambridge University Press.

Hajer, M., \& Laws, D. (2006). Ordering through discourse. En M. Moran, M. Rein \& R. E. Goodin (Eds), The Oxford handbook of public policy (pp. 251-268). Oxford: Oxford University Press.

Hajer, M., \& Wagenaar, H. (Eds.). (2003). Deliberative policy analysis. Understanding governance in the network society. Cambridge: Cambridge University Press.

Kay, A. (2009). Understanding policy change as a hermeneutic problem. Journal of Comparative Policy Analysis: Research and Practice, 11(1), 47-63.

Krause, M. (2002). The institutionalization of community interventions in Chile: characteristic and contradictions. American Journal of Community Psychology, 30(4), 554-570.

Lapalma, A., \& De Lellis, M. (2012). Psicología comunitaria y políticas públicas. Una articulación posible y necesaria. En A. Sánchez, J. Alfaro \& A. Zambrano (Eds.), Psicología comunitaria y políticas 
sociales: reflexiones y experiencias (pp. 147-172). Buenos Aires: Paidós.

Marcoux, H., Angelique, C., \& Culley, M. R. (2009). La psychologie communautaire en Amérique du Nord: Historiques et applications. Pratiques Psychologiques, 15(1), 17-27.

Montero, M. (2010). Unión de actores sociales, participación comunitaria y ética en la ejecución de políticas públicas. En A. Hincapié (Comp.), Sujetos políticos y acción comunitaria. Claves para una praxis de la psicología social y de la clínica social-comunitaria en América Latina (pp. 85-114). Medellín: Universidad Pontificia Bolivariana.

Nelson, B. (2001). Políticas públicas y administración: una visión general. En R. Goodin \& H. D. Klingemann (Eds.), Nuevo manual de ciencia política (pp. 795-860). Madrid: Istmo.

Ornelas, J., Vargas-Moniz, M., \& Madeira, T. (2012). Contribuciones de la psicología comunitaria a las políticas sociales en las áreas de la integración psicosocial y en la violencia contra las mujeres. En A. Sánchez, J. Alfaro \& A. Zambrano (Eds.), Psicología comunitaria y políticas sociales: reflexiones y experiencias (pp. 255-288). Buenos Aires: Paidós.

Perkins, D. (1995). Speaking truth to power: Empowerment ideology as social intervention and policy. American Journal of Community Psychology, 23(5), 730-765.

Phillips, D. (2000). Social policy and community psychology. En J. Rappaport \& E. Seidman (Eds.), Handbook of community psychology (pp. 397-420). Nueva York: Kluwer Academic-Plenum Publishers.

Rappaport, J. (1977). Community psychology: Values, research, and action. Nueva York: Holt, Rinehart $\&$ Winston.

Rein, M., \& Schön, D. A. (1993). Reframing policy discourse. En F. Fischer \& J. Forester (Eds.), The argumentative turn in policy analysis and planning (145-166). Durham, NC: Duke University Press.

Rodríguez, A. (2009). Social policies in Uruguay: A view from the political dimension of community psychology. American Journal of Community Psychology, 43(1-2), 122-133.
Sabatier, P., \& Jenkins-Smith, H. (1993). Policy change and learning: An advocacy coalition approach. Boulder, CO: Westview Press.

Schlager, E. (1999). A comparison of frameworks, theories and models of then policy process. En P. A. Sabatier (Ed.), Theories of the policy process (pp. 233-260). Boulder, CO: Westview Press.

Solarz, A. (2001). Investing in children, families, and communities: Challenges for an interdivisional public policy collaboration. American Journal of Community Psychology, 29(1), 1-14.

Shinn, M. (2007). Waltzing with a monster: Bringing research to bear on public policy. Journal of Social Issues, 63(1), 215-231.

Schön, D. A., \& Rein, M. (1994) Frame reflection: Toward a resolution of intractable policy controversies. New York: Basic Books.

Schmidt, V. (agosto, 2006). Bringing the State back into the varieties of capitalism and discourse back into the explanation of change. Trabajo presentado en The National Meetings of the American Political Science Association, Philadelphia, PA.

Stone, D. (2002). Policy paradox and political reason. Nueva York: Norton.

Teixeira, P., Loja, E., Costa, E. \& Menezes, I. (2012). Más allá del desierto: Empoderamiento e inclusión en las políticas sociales en el dominio de la rehabilitación en Portugal y en Europa. Portugal: Paidós

Vlassopoulou, C. A. (1999). La lutte contre la pollution atmosphérique urbaine en France et en Grèce. Construction des problèmes publiques et changement de politique (Tesis doctoral inédita). Université Panthéon ASSAS, Paris II.

Wildavsky, A. (1969). Rescuing policy analysis from PPBS. Public Administration Review, 29(2), 189-202.

Zimmerman, M. A., \& Rappaport, J. (1988). Citizen participation, perceived control, and psychological empowerment. American Journal of Community Psychology, 16(5), 725-750.

Zittoun, P. (2009). Understanding policy change as a discursive problem. Journal of Comparative Policy Analysis, 11(1), 65-82. 
